\title{
Lessons learnt from network modelling in a low heat density district heating system
}

\author{
Itzal del Hoyo Arce Susana López Perez Saioa Herrero López Iván Mesonero Dávila \\ IK4-TEKNIKER \\ Parke Teknologikoa, Iñaki Goenaga 5, 20600 Eibar (Spain) \\ \{itzal.delhoyo, susana.lopez, saioa.herrero, ivan.mesonero\}@tekniker.es
}

\begin{abstract}
This paper presents the lessons learnt during the development of a library for the modelling of district heating systems (DH systems), especially focusing on the distribution network. The library was built based on elements from the Modelica Standard Library (Modelica Association, 2012) and the NewThermal library (Lopez, del Hoyo, 2014).

The modelling strategy chosen is described. Furthermore, the requirements established by the DH networks are set out as well as the models created in response to these demands.

Finally, the artificial diffusion phenomenon, present in the simulation of this kind of thermo-fluid systems, is described.
\end{abstract}

Keywords: $\quad$ district heating modelling, plastic pipe model, buried pipe model, numerical diffusion, courant number, transport delay, thermo-fluid simulation

\section{Introduction}

District heating (DH) systems produce hot water (or steam) at a central plant. The hot water is then transported through pipes placed underground to individual buildings for space heating or domestic hot water (DHW) generation. Therefore, dwellings in a DH system do not need their own boiler or any other generation system. Hence, a DH system is composed of a generation system, distribution system and consumption side.

The main objective of modelling DH networks is to simulate the rate of energy transport through the system. This transport depends on the water flow through the system as well as on the temperature levels in the DH network. Therefore, as well as in the case of the modelling of other large scale thermo-fluid systems, in the modelling of a DH system there are also fundamentally two different dynamics to take into consideration, flow and temperature dynamics (Frederiksen, Werner, 2013). The most important difference between them is that while changes in the flow are quickly transferred to the whole network, usually in a matter of seconds, temperature changes are transferred relatively slowly, in some cases taking several hours.
The temperature dynamics are therefore the main dynamics in a DH system and it is essential to consider them. In the case of the hydraulic dynamics, the debate is not so clear. The majority of authors work with pseudo-dynamic models, in which the flow and the pressure are calculated based on a static flow model, because in most cases hydraulic dynamics are presumably irrelevant. However, the advantages of a variable flow in a DH system are the low return temperature and the low heat losses in the network. The disadvantage, however, is the risk of insufficient hydraulic balance (Boysen et al, 2007). In this case, the dynamic hydraulic balance is key to the automatic control of the flow, so if the systems require a dynamic balance, the hydraulic dynamics of the system have to be taken into account.

In the framework of FP7 European project AMBASSADOR (Autonomous Management System Developed for Building and District Levels) led by Schneider Electrics (Onillon, 2014), the dynamic model library for the modelling of DH systems was carried out under Modelica ${ }^{\circledR}$. The library is composed of models for the generation system (such as boilers or solar thermal collectors) and distribution system (such as pipes, fluid or hydraulic balance valves). The objective of the AMBASSADOR project is the development of and experimentation with systems and tools that aim to optimize the energy usage within a district by managing energy flows, and predicting and mastering energy consumption and energy production. In the case of District Heating systems, control design requires knowing in detail the physical behaviour of the system to be controlled. A library containing detailed models of the components present in a DH system was developed in consequence, including the DH network system on which this paper is focused.

Within this frame, a fully dynamic modelling has been chosen for the modelling of the distribution network, where both the temperature and the flow are simulated dynamically. The modelling is based on Modelica ${ }^{\circledR}$ and uses a Dymola ${ }^{\circledR}$ environment due to the advantages offered (Basciotti, 2012), such as the possibility to implement customised control strategies or the possibility to consider stationary effects and dynamic hydraulic phenomena. 
The lessons learnt during the modelling of a low heat density DH network and some of the models developed, as well as the limitations detected, are presented below.

\section{Modelling of DH networks}

The main components in a DH network, that is, the distribution system, are the medium that contains the energy to be distributed and the pipes through which the water flows through the network. The models described below are focused on these main components, although during the AMBASSADOR project other models, such as the hydraulic balance valve or DH substations, also present in the network, were developed.

\subsection{Medium: AdaptableSimpleWater model}

The Modelica Standard Library contains different Water models depending on the characteristics taken into account: compressibility, properties variation with temperature, etc. Starting from the most basic ConstantPropertyLiquidWater model (which considers the liquid incompressible and its properties constant), to a more sophisticated set of WaterIF 97 models (which take into account compressible fluid with properties dependent on temperature).

The more real approximation implies using the type of water that takes into account both the compressibility and the properties dependent on temperature. However, this kind of simulations have a high computation time and are usually difficult to initialize.

Fluid operation range in $\mathrm{DH}$ systems is usually around $30^{\circ} \mathrm{C}-110^{\circ} \mathrm{C}$ (Frederiksen,Werner, 2013) so if a ConstantPropertyLiquidWater model is used significant errors in pressure loss calculation and thus in mass flow rate values could appear. Since although water is essentially incompressible, especially under normal conditions, mass flow rate calculation usually implies the value of fluid viscosity, which depends on temperature. Therefore, a certain error will be involved if constant thermal properties are considered in the fluid model.

With the aim of quantifying the error made, three different water models have been studied:

- RealWater model (WaterIF97_ph): compressible fluid with properties dependent on temperature. Available in the Modelica Standard Library.

- SimpleWater model (ConstantPropertyLiquid): incompressible fluid with constant properties established at $20^{\circ} \mathrm{C}$. Available in the Modelica Standard Library.

- SimpleWater70C model (ConstantPropertyLiquid70C): incompressible fluid with constant properties established at $70^{\circ} \mathrm{C}$, which is the operation temperature midrange of the $\mathrm{DH}$ network modelled. Created for the study.
The comparison has been carried out for two kinds of pipes involved in the DH network,

- A flexible plastic pipe for low-temperature application (DN50) present in the transmission piping

- An usual copper pipe (28mm external diameter) covered by a specific insulation, present in the $\mathrm{DH}$ branches

For both analysis, a $10 \mathrm{~m}$ pipe has been considered and two different mass flow rate values have been used, specifically, the extreme values of the real system $(0.016 \mathrm{~kg} / \mathrm{s}$ and $0.25 \mathrm{~kg} / \mathrm{s}$ in the case of copper pipe, and $0.25 \mathrm{~kg} / \mathrm{s}$ and $2 \mathrm{~kg} / \mathrm{s}$ for the flexible pipe). In addition:

- No heat transfer with the ambient has been considered, just $10^{\circ} \mathrm{C}$ were imposed in the most external layer of the pipes.

- An inlet temperature ramp between $80^{\circ} \mathrm{C}$ and $60^{\circ} \mathrm{C}$ (in 2000s) has been simulated.

The pressure drop of both pipes were analysed during the experiments. For the calculation, the "DetailedPipeFlow" option has been chosen for the FlowModel replaceable model, in this model, the wall friction in laminar and turbulent regimes is considered.

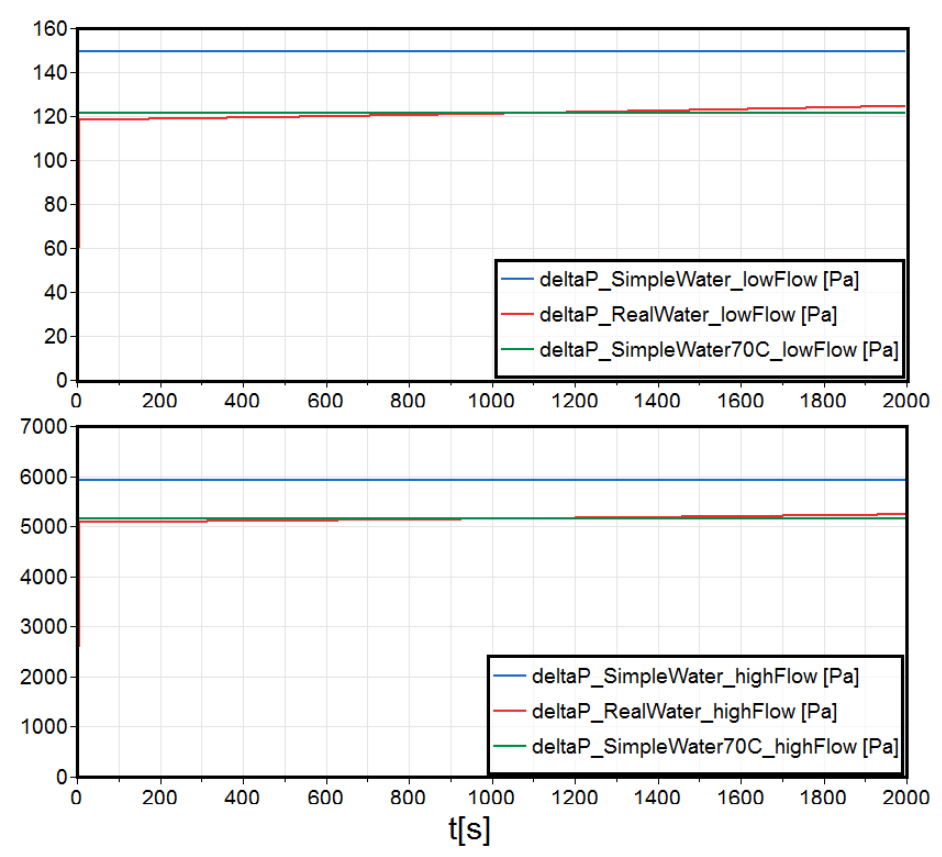

Figure 1. Pressure drop $[\mathrm{Pa}]$ in copper pipe (top: $0.016 \mathrm{~kg} / \mathrm{s}$, bottom: $0.25 \mathrm{~kg} / \mathrm{s}$ ) 

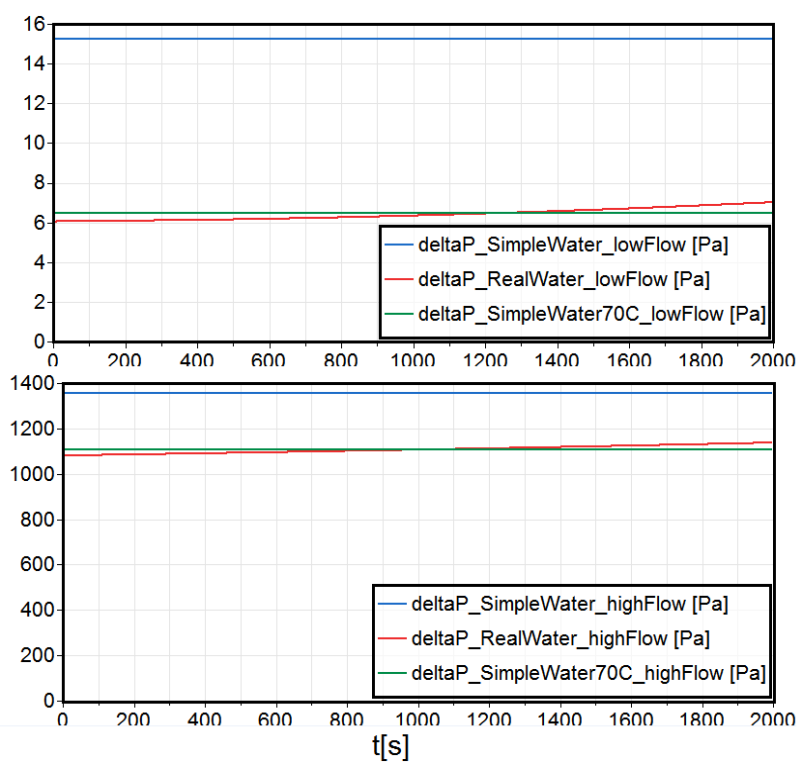

Figure 2. Pressure drop [Pa] in flexible plastic pipe (top: $0.25 \mathrm{~kg} / \mathrm{s}$, bottom: $2 \mathrm{~kg} / \mathrm{s}$ )

In Figure 1 and Figure 2 the pressure drop values obtained in the simulations are shown. As can be observed, the pressure loss value obtained with SimpleWater70C (in green) is closer to the RealWater mean value (in red) than the SimpleWater (in blue), thus, giving a more realistic behaviour. In addition, the model with RealWater, is 10 times slower than the others.

As expected, RealWater is the only one that shows the pressure loss variation with temperature, as the properties of the other two fluids do not depend on temperature. However, it is observed that the variation with respect to the mean value is very low, so a constant temperature fluid approximation does not seem to introduce a relevant error for the cases, such as the DH system at hand, where the operating temperature range is not very wide.

The comparison has been done considering the RealWater model's results as those that are nearer to the real system's results.

Table 1. Maximum relative error in pressure drop compared with RealWater in copper and flexible pipes

\begin{tabular}{|c|c|c|c|c|}
\hline \multirow{2}{*}{ Maximum error } & \multicolumn{2}{|c|}{ Flexible pipe } & \multicolumn{2}{c|}{ Copper pipe } \\
\cline { 2 - 5 }$[\%]$ & Low & High & Low & High \\
& Flow & Flow & Flow & Flow \\
\hline SimpleWater & 26.44 & 16.6 & 51.9 & 25.19 \\
\hline SimpleWater70C & 2.54 & 1.4 & 6.77 & 2.38 \\
\hline
\end{tabular}

Table 1 shows the high error made with the SimpleWater model in the calculation of pressure drop in comparison with the RealWater results. In the case of copper pipe, in addition, the error made is higher because the flow is on all occasion laminar or near laminar.
Besides, additional simulations have been run for RealWater and SimpleWater70C, but this time the pipe outlet temperature differences have been compared. Once again, simulations have been run for both the copper and the flexible plastic pipes, forcing a transient phase and imposing $\mathrm{T}=10^{\circ} \mathrm{C}$ in the last layer of the pipes.

In the case of the flexible pipe, present in the transmission piping of the $\mathrm{DH}$ network and therefore more suitable to major changes in mass flow and different levels of temperature, a change in flow (from $0.03 \mathrm{~kg} / \mathrm{s}$ to $2 \mathrm{~kg} / \mathrm{s}$ ) has been imposed and different input temperatures have been tested for a $200 \mathrm{~m}$ pipe. Three inlet temperatures were considered:

- $\quad$ InletTemperature $1=70^{\circ} \mathrm{C}$

- InletTemperature $2=85^{\circ} \mathrm{C}$

- $\quad$ InletTemperature $3=95^{\circ} \mathrm{C}$

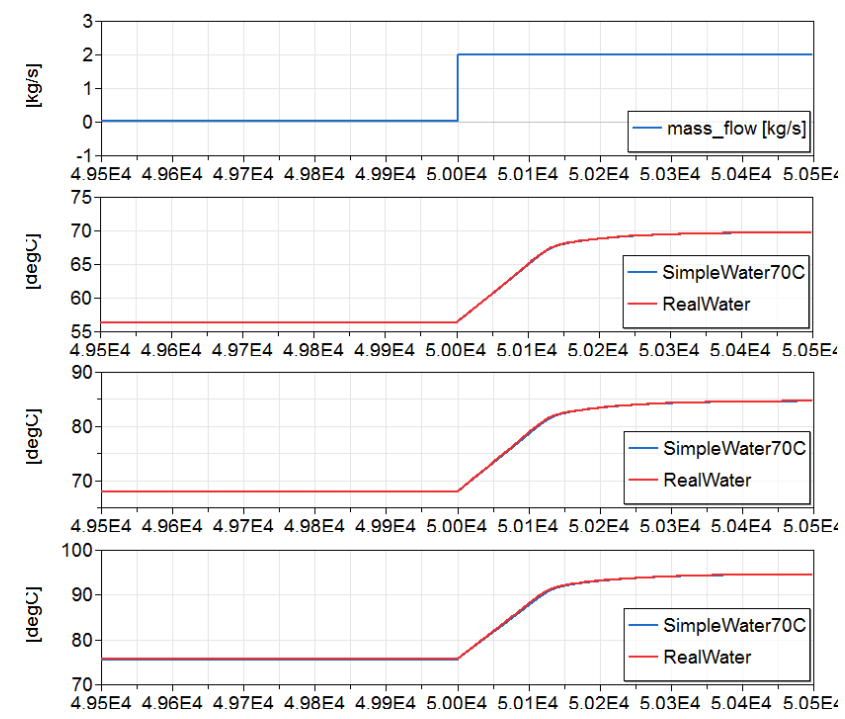

Figure 3. Output temperature differences in a $200 \mathrm{~m}$ plastic pipe, under a mass flow rate step and coming from three different input temperatures

Figure 3 shows little differences in the output temperature in all the experiments.

Table 2. Outlet temperature comparison. Errors made by SimpleWater70C model compared with RealWater model's results

\begin{tabular}{|c|c|c|c|}
\hline $\begin{array}{c}\text { Inlet } \\
\text { temperature } \\
{\left[{ }^{\circ} \mathrm{C}\right]}\end{array}$ & $\begin{array}{c}\text { Abs error } \\
{\left[{ }^{\circ} \mathrm{C}\right]}\end{array}$ & $\begin{array}{c}\text { Mean } \\
\text { temperature } \\
{\left[{ }^{\circ} \mathrm{C}\right]}\end{array}$ & $\begin{array}{c}\text { Rel. Error } \\
{[\%]}\end{array}$ \\
\hline 70 & 0.18 & 63 & 0.3 \\
\hline 85 & 0.334 & 76 & 0.44 \\
\hline 95 & 0.474 & 85 & 0.56 \\
\hline
\end{tabular}

In the case of copper pipe, a constant mass flow has been considered and a temperature step (from $70^{\circ} \mathrm{C}$ to $75^{\circ} \mathrm{C}$ ) has been imposed in the inlet temperature. 

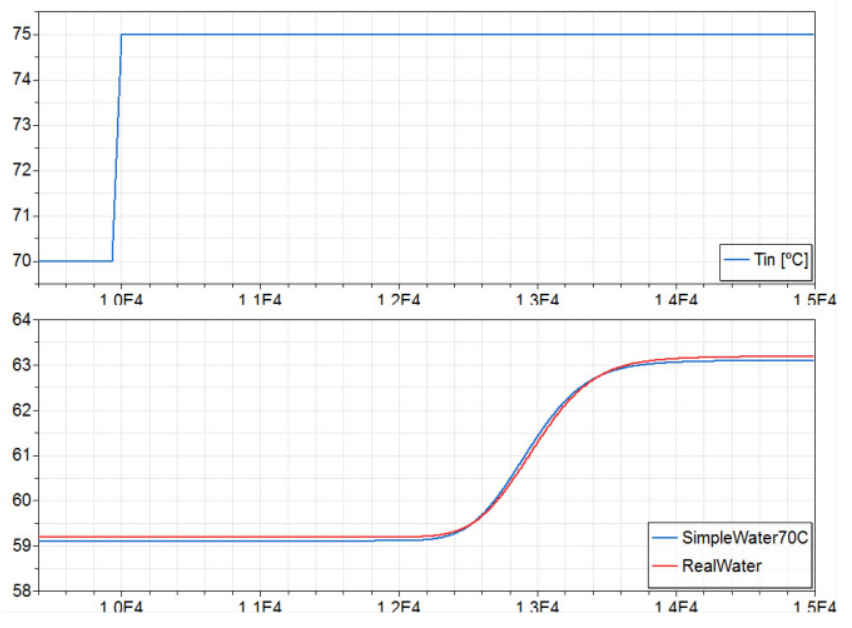

Figure 4. Output temperature in an $80 \mathrm{~m}$ copper pipe under inlet temperature step and constant mass flow rate (Real Water vs. SimpleWater70C)

In this case, the maximum absolute error is $0.217^{\circ} \mathrm{C}$, which, compared to the mean temperature value $\left(72.5^{\circ} \mathrm{C}\right)$, results in a maximum relative error of $0.3 \%$.

A similar analysis has been carried out for different applications, such as a little flat plate solar collector plant for generating domestic hot water (DHW), with the same conclusion: it is considered acceptable to use an incompressible and temperature independent fluid, with properties established at operation temperature midrange.

Although the use of a fluid with properties established at operation temperature midrange is not always applicable, in many cases, as above explained, it is a useful simplification. Nevertheless, the calculation of the maximum error made with this simplification is, on all occasions, crucial. Consequently, a new water model has been developed, adaptableSimpleWater model. This water model considers water incompressible and that it has properties of the fluid independent of temperature. However, the adaptablesimplewater model calls for the midrange operation temperature of the system to be simulated, and during the whole simulation, it uses the properties of the fluid relative to this established temperature.

Hence, the model has the properties of the fluid declared as a function of the midrange operation temperature. This temperature is established when the adaptablesimplewater class is instantiated. Both, the properties of the fluid and the midrange operation temperature, are declared as a constant.

\subsection{Distribution pipes: InsulatedPipe model}

The level of detail concerning temperature dynamics, especially the inclusion of the heat capacity of the insulation material and soil, is often omitted in simulations of large scale district heating systems.
These systems also have considerable heat demand and high flow rates resulting in reasonably stable temperature levels within the distribution network. In local small scale and low heat density systems $(<0.5$ $\mathrm{MWh} / \mathrm{m}$ ), the behaviour of a single consumer is more important thus making a detailed modelling, with smaller time steps being more relevant. This need is further increased in a hybrid system with alternative sources of heat at different temperatures and that are intermittently available, e.g. solar heat. Systems with low heat demand experience significant fluctuations in temperature especially within the connection pipes, i.e. pipes connecting consumers to the distribution network.

While a less detailed model can give adequately accurate results for low heat demand systems on a yearly level, e.g. for heat losses, they are less useful in testing control systems in different use cases and can lead to systems that do not operate as they were designed to according to the simulations.

During the AMBASSADOR project, the dynamic modelling of a low heat density DH network was carried out with control design purpose, therefore, detailed models of distribution pipes were developed considering the heat capacity of all materials present in the pipe. Different versions of the insulatedPipe model (Lopez, del Hoyo, 2014) were used as a basis.

The insulatedPipe basic model describes the hydraulic and thermal behaviour of any pipe with one or more solid layer(s) assuming radial symmetry in both phenomena. The DH network in this case, however, requires new features in the insulatedPipe model, so the basic model has been expanded creating new versions.

\subsubsection{Neglecting axial heat transmission}

It is common to neglect the axial heat transmission throughout the length of the cover in the district heating and cooling systems pipes (DHC systems). It is done in the most used methods for the modelling of DHC systems, the node method and element method (Pálsson, 2000). This happens because considering the slow temperature dynamics and the poor conductor plastic materials in DHC pipes, the heat transfer in the solid materials of the pipe principally occurs in the radial direction. Traditionally, in the case of modelling DHC systems, there are no major differences between the results of taking into account the axial heat transfer and not taking it into account, but there is a big difference regarding simulation time. Not considering the heat transfer in axial direction reduces considerably computational weight of the simulation, a huge advantage in the simulation of large-scale thermo-fluid systems, because it can significantly speed up simulations.

Hence, based on state of the art, an improved InsulatedPipe model was created, called the InsulatedPipeoptionalAxial model. This 
new model has the same characteristics as the InsulatedPipe basic model, but it has the option of choosing between taking into account the heat transfer in axial direction or not considering it. Thus, it is up to the user to decide regarding the aforementioned heat transfer. When the axial heat transfer is neglected the boolean axial Heat has to be switched to false.

The experiments done show practically identical results. During the experiment, the pipes were considered adiabatic and they were fed by a pump with a constant mass flow $(2 \mathrm{~kg} / \mathrm{s})$ and a ramp in temperature (from $20^{\circ} \mathrm{C}$ to $80^{\circ} \mathrm{C}$, in $100 \mathrm{~s}$ ). In the Figure 6 , the temperatures of three nodes (the first node, the middle node and the last node) are shown for the cases where axial heat conduction is considered and neglected under the same conditions.
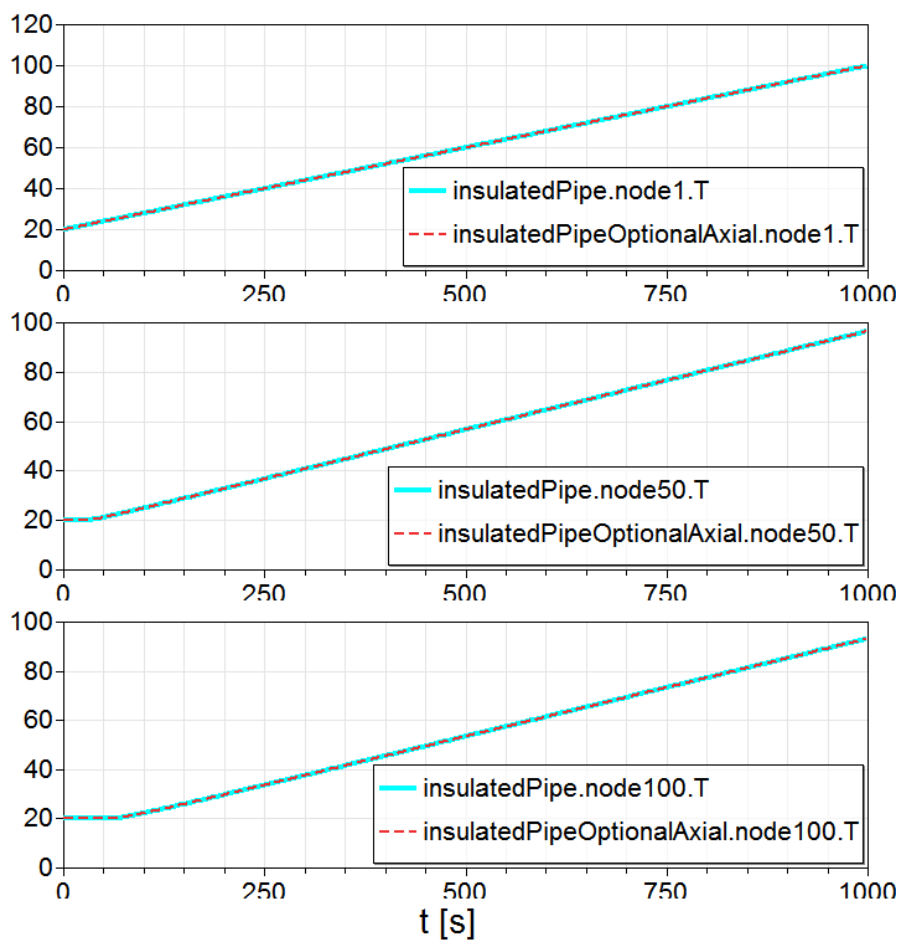

Figure 5. Temperature of three nodes inside the InsulatedPipe and InsulatedPipeoptionalAxial models

The CPU time consumed, nevertheless, varies significantly, since neglecting the axial heat conduction speeds up the simulation by a factor of 2.69 (the simulation neglecting axial heat conduction can be up to $60 \%$ faster).

\subsubsection{Buried pipe}

The pipes used for transmission in DH systems are usually underground. Buried systems are highly influenced by the soil around them, both the thermal conductivity of the ground and the depth at which they are buried affects the heat transfer in the system, particularly when the insulation has low thermal resistance (McCauley, 2000). Moreover, soil thermal conductivity changes significantly with moisture content, from $0.14 \mathrm{~W} / \mathrm{mK}$ in dry soil conditions to 2.16 $\mathrm{W} / \mathrm{mK}$ in wet soil conditions (Bottorf, 1951).

The most important factors affecting heat transfer are the difference between earth and fluid temperatures and the thermal insulation. Other factors that affect heat transfer are (ASHRAE handbook, 2008):

1. Depth of burial, related to the earth temperature and soil thermal resistance

2. Soil conductivity, related to soil moisture content and density

3. Distance between adjacent pipes.

The mathematical model of DHC system pipes must compute transient heat gains or losses in the underground piping system, and for this, the resistance of the ground has to be considered. The most usual physical model defines thermal resistances between the different materials of the pipe and surrounding ground. That is, in the modelling of a buried pipe, it is divided into three main parts, the surrounding ground, the insulation layers and the water mass.

The surrounding ground is considered, as an infinite inertia. That is, it is supposed that the heat from the buried pipes is not enough to change the temperature and thermal properties of the surrounding soil. Therefore, the influence of the surrounding soil is taken into consideration through a thermal resistance $\left(\mathrm{R}_{\mathrm{g}}\right)$, depending on the depth at which the pipe is buried $\left(\mathrm{s}_{\mathrm{d}}\right)$, the conductivity of the ground $\left(\mathrm{K}_{\mathrm{g}}\right)$ and the external diameter of the buried pipe $\left(D_{m}\right)$, as is shown in the following equations (Pálsson, Larsen, et al, 1999):

$$
\begin{gathered}
R_{g}=\frac{1}{2 \pi K_{g}} \ln \left(\frac{4 H}{D_{m}}\right) \\
H=s_{d}+0.0685 K_{g}
\end{gathered}
$$

A second thermal resistance $\left(\mathrm{R}_{\mathrm{H}}\right)$ is presented in the pipe model to take into account the effect of having two pipes side by side in the ground, depending on the depth at which the pipes are buried $\left(\mathrm{s}_{\mathrm{d}}\right)$, the conductivity of the ground $\left(\mathrm{K}_{\mathrm{g}}\right)$ and the distance between the centre of the two buried pipes $\left(\mathrm{s}_{\mathrm{c}}\right)$. Assuming identical supply and return pipes, this resistance is given as (Pálsson, Larsen, et al, 1999):

$$
R_{H}=\frac{1}{2 \pi K_{g}} * \ln \left(1+\left(\frac{2 H}{s_{c}}\right)^{2}\right)
$$

The buried model has been used for the modelling of a CALPEX ${ }^{\circledR}$ district heating pipe and the validation has been done with the data provided by the manufacturer (CALPEX® technical sheet). The sheet shows two CALPEX®UNO $63 / 126$ buried at $0.60 \mathrm{~m}$ at a distance 
of $0.1 \mathrm{~m}$. The ground temperature and soil conductivity are also known:

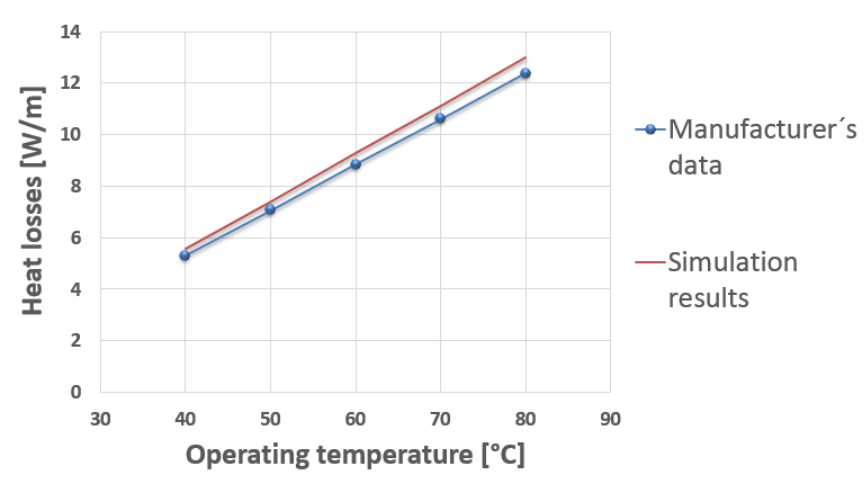

Figure 7. Validation of insulated and buried pipe model against data provided by the manufacturer

The error of the simulation results compared with those provided by the manufacturer is $5.01 \%$ at highest water temperature (distribution temperature) and is below the latter value at lower temperatures.

\section{Discussion: Artificial diffusion phenomena}

During the process of modelling the DH network, a well-known phenomenon has been detected in the simulation results related with the transport delay which arises in any fluid movement through a pipe. The real transport delay is calculated in the following way:

$$
t_{\text {delay }}=\frac{\rho * A * L}{Q}
$$

In the equation (4), $\rho$ is the density of the fluid, $\mathrm{A}$ is the transverse area the fluid goes through, $\mathrm{L}$ is the length at which the output temperature is observed and Q the flow rate through the system.

In the course of the validation period of the insulatedPipe model, the following test was suggested for a flexible plastic pipe of $100 \mathrm{~m}$ length, divided into 10 nodes:

- Mass flow: Step signal, the mass flow changes from $1 \mathrm{~kg} / \mathrm{s}$ to $2 \mathrm{~kg} / \mathrm{s}$ at the 30 th minute

- Temperature of the incoming water: Double step signal, the incoming water temperature changes from $80^{\circ} \mathrm{C}$ to $70^{\circ} \mathrm{C}$ at the 20 th minute and again to $60^{\circ} \mathrm{C}$ at the 40 th minute

- It is assumed the ground temperature remains constant $\left(10^{\circ} \mathrm{C}\right)$ during the simulation

\section{Outlet Temperature $\left[{ }^{\circ} \mathrm{C}\right]$}

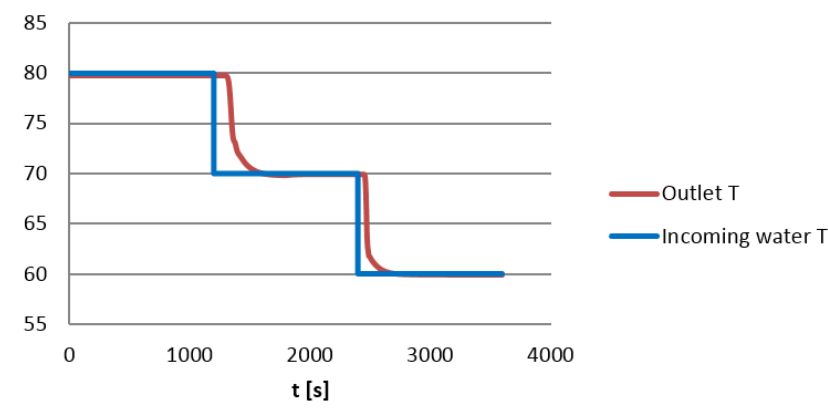

Figure 8. Outlet temperature in a $100 \mathrm{~m}$ pipe divided into 10 nodes

The real transport delays, calculated by equation (4), that must appear on the outlet temperature behaviour corresponding to the first temperature step and the second temperature step are $130.5 \mathrm{~s}$ and $65.3 \mathrm{~s}$ respectively. However, the transport delays in the dynamic response of the model are, rounding, 60s after the first step and $35 \mathrm{~s}$ in the second one. This phenomenon is known as artificial diffusion.

Artificial diffusion is the consequence of numerical diffusion in the simulation of a continuum phenomenon such as fluid movement, in consequence, the simulated medium exhibits a higher diffusivity than the true medium (Leveque, 2007). In this case, the diffusivity is known as the property of a substance indicative of the rate at which a thermal disturbance, such as a drop in temperature, will be transmitted through the substance. Therefore, the model shows a higher diffusivity than the real case, that is, the drop in temperature through the substance is transmitted faster than in the real case.

The numerical diffusion is often analysed taking the Courant number into account:

$$
\operatorname{Cou}=\frac{\Delta t * u}{\Delta x}
$$

When the Courant number approaches zero the model shows excessive artificial diffusion while $\mathrm{Cou}=1$ cases give the exact result.

The Courant number depends on the time step, that is, the time between the current and the previous step $(\Delta \mathrm{t})$, the element length $(\Delta \mathrm{x})$ and the flow velocity $(\mathrm{u})$. The latter is imposed by the real case, and in the case of using a variable time-step solver, in addition, in the vast majority of cases, the software itself decides the timestep value $(\Delta t)$. The integration step size in variable time-step solvers, is chosen in such a way, that the local error is smaller than the desired maximum local error, defined via the relative and absolute tolerances. In other words, a variable (or adaptive) step size implies that the algorithm adapts the step size to meet a local error criterion based on the tolerance (Dassault Systèmes AB. Dymola User Manual, 2015). 
Therefore, the user can specify just the element length; accordingly, the artificial diffusion could be improved discretizing more the pipe element. Therefore, in the case at hand, the higher the number of nodes in the pipe the better the results of the model. Considering three different degrees of discretization:

- Considering the pipe divided into one node: $\Delta \mathrm{x}=100 \mathrm{~m}$

- Considering the pipe divided into 10 nodes: $\Delta \mathrm{x}=10 \mathrm{~m}$

- Considering the pipe divided into 100 nodes: $\Delta \mathrm{x}=1 \mathrm{~m}$

The simulation has been carried out with the same tolerance and the exercise's results are shown in the following figure:

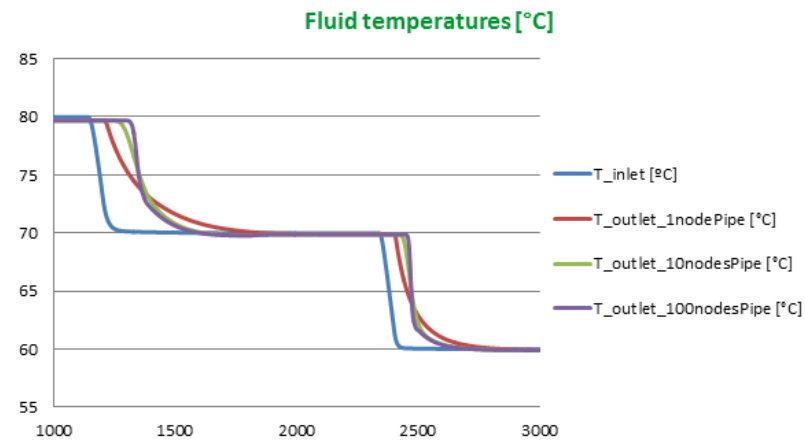

Figure 9. Outlet water temperature for the cases of the pipe discretized into one node, into 10 nodes and into 100 nodes

Figure 9 demonstrates that the fewer the nodes in the pipe the higher the artificial diffusion.

Table 3. Courant number for each case

\begin{tabular}{|l|c|c|c|}
\hline $\begin{array}{c}\text { Mass flow } \\
1 \mathrm{~kg} / \mathrm{s}\end{array}$ & $\begin{array}{c}\text { l node } \\
\Delta x=100 \mathrm{~m}\end{array}$ & $\begin{array}{c}\text { 10 nodes } \\
\Delta x=10 \mathrm{~m}\end{array}$ & $\begin{array}{c}100 \text { nodes } \\
\Delta x=1 \mathrm{~m}\end{array}$ \\
\hline $\begin{array}{l}\text { Delay in the } \\
\text { model [s] }\end{array}$ & 35 & 65 & 112 \\
\hline $\begin{array}{l}\text { Relative } \\
\text { error in the } \\
\text { time delay } \\
{[\%]}\end{array}$ & 73 & 50 & 14 \\
\hline Cou number & 0.007 & 0.068 & 0.75 \\
\hline $\begin{array}{c}\text { Mass flow } \\
2 \mathrm{~kg} / \mathrm{s}\end{array}$ & $\begin{array}{c}1 \text { node } \\
\Delta x=100 \mathrm{~m}\end{array}$ & $\begin{array}{c}10 \text { nodes } \\
\Delta x=10 \mathrm{~m}\end{array}$ & $\begin{array}{c}100 \text { nodes } \\
\Delta x=1 \mathrm{~m}\end{array}$ \\
\hline Cou number & 0.015 & 0.138 & 1.517 \\
\hline
\end{tabular}

In Table 3 can be seen that the Courant number changes with the number of nodes in the pipe, but as is known intuitively with the case of the 100 nodes pipe (last column), the adequate number of nodes for a specific operating flow rate may not be the best one for other operating flow rate. Hence, the degree of discretization must be in accordance with the operating flow rate range. In addition, when the discretizing level increases the tolerance of the integration has to change accordingly, otherwise, during the calculation a numerical error may appear which is reflected in the results as artificial diffusion (that is, a thermal disturbance is transmitted through the substance faster than in the real case).

The major disadvantage of this procedure to get the best discretization level of a big thermo-hydraulic system in order to reduce the relevance of the artificial diffusion, is the significant time needed. Since according to the technical support service, using a variable time-step solver is not possible to examine the time step during the course of the simulation. Therefore, the methodology requires running the simulation to have available the necessary data for the calculation of the Courant number. This kind of thermo-fluid simulation, moreover, usually has a high computational weight.

\section{Conclusion}

The models of the two main components present in a DH network, fluid and buried pipes, have been developed and successfully validated. These models have been developed with elements from both the Modelica Standard Library and NewThermal library (which is in turn based on models from the Modelica Standard Library), and based on the requirements of district heating systems.

Furthermore, the artificial diffusion phenomenon detected in the network models is explained and it is suggested to identify and control its influence through the calculation of the Courant number. The analysis of the Courant number, however, now implies a postprocessing of the results since it is not possible to evaluate the time step during the course of the simulation using a variable time-step solver.

\section{Acknowledgements}

The research leading to the results presented in this paper (models development, analysis, validation, etc.) has been developed in the framework of project AMBASSADOR, which has received funding from the European Union Seventh Framework Programme [FP7/2007-2013] under grant agreement n³14175

\section{References}

ASHRAE Handbook: Heating, Ventilating \& AirConditioning Systems, chapter 11. 2008

Basciotti D., Pol O., A theoretical study of the impact of using small scale thermo chemical storage units in district heating networks. Energy Department AIT, Austria (2012)

Bottorf, J.D. Summary of thermal conductivity as a function of moisture. 1951

Boysen, Thorsen. Technical paper, Hydraulic balance in a district heating system (2007)

Dassault Systèmes AB. Dymola User Manual (2015)

Svend Frederiksen, Seven Werner. District Heating and Cooling. Studentlitteratur first edition (2013) 
R.J. Leveque (2007). Finite Difference Methods for Ordinary and Partial Differential Equations. ISBN 978-0-898716-290

Susana López, Itzal del Hoyo. Proposal for standardization of Heat Transfer Modelling in NewThermal Library. $10^{\text {th }}$ International Modelica Conference (Lund), 2014.

James McCauley. Steam distribution system deskbook (ISBN 0-88173-303-2), 2000

Modelica Association, (2012). A Unified Object-Oriented Language for Physical System Modeling, Modelica ${ }^{\circledR}$

Emmanuel Onillon, District energy flow optimization taking into account building flexibilities. International Conference Sustainable Places (2014)

Halldór Pálsson. Methods for Planning and Operating Decentralized Combined Heat and Power Plants. Riso National Laboratory, Roskilde, Technical University if Denmark, 2000

Halldór Pálsson, Helge V. Larsen, et al. Equivalent models of district heating system, Technical University of Denmark and Risø National Laboratory, 1999. 